\title{
pMAA-Red: a new pPZP-derived vector for fast visual screening of transgenic Arabidopsis plants at the seed stage
}

Muhammad Amjad Ali ${ }^{1 \dagger}$, Kausar Hussain Shah ${ }^{1 \dagger}$ and Holger Bohlmann ${ }^{1,2^{*}}$

\begin{abstract}
Background: The production of transgenic plants, either for the overproduction of the protein of interest, for promoter: reporter lines, or for the downregulation of genes is an important prerequisite in modern plant research but is also very time-consuming.

Results: We have produced additions to the PPZP family of vectors. Vector PPZP500 (derived from PPZP200) is devoid of Notl sites and vector pPZP600 (derived from pPZP500) contains a bacterial kanamycin resistance gene. Vector pMAA-Red contains a Pdf2.1: DsRed marker and a CaMV:: GUS cassette within the T-DNA and is useful for the production of promoter: GUS lines and overexpression lines. The Pdf2.1 promoter is expressed in seeds and syncytia induced by the beet cyst nematode Heterodera schachti in Arabidopsis roots. Transgenic seeds show red fluorescence which can be used for selection and the fluorescence level is indicative of the expression level of the transgene. The advantage is that plants can be grown on soil and that expression of the marker can be directly screened at the seed stage which saves time and resources. Due to the expression of the Pdf2.1: DsRed marker in syncytia, the vector is especially useful for the expression of a gene of interest in syncytia.
\end{abstract}

Conclusions: The vector pMAA-Red allows for fast and easy production of transgenic Arabidopsis plants with a strong expression level of the gene of interest.

Keywords: Transient expression, pPZP family vectors, Marker gene, Agroinfiltration, DsRed, Agrobacterium, Arabidopsis transformation

\section{Background}

Modern plant research relies heavily on the use of transient and stable transformation with the help of Agrobacterium tumefaciens [1,2]. This bacterium is a natural genetic engineer and transfers a part of its large Ti plasmid into host plants to induce cell division and the synthesis of opines. For use in genetic engineering, the Ti plasmid has been divided into a helper plasmid which is devoid of the T-DNA and remains within the Agrobacteria and a binary vector carrying the T-DNA which can be manipulated in E. coli. The first widely used binary vector was pBIN19

\footnotetext{
* Correspondence: holger.bohlmann@boku.ac.at

${ }^{\dagger}$ Equal contributors

'Division of Plant Protection, Department of Crop Sciences, University of Natural Resources and Life Sciences, Vienna, Austria

${ }^{2}$ Division of Plant Protection, Department of Crop Sciences, University of Natural Resources and Life Sciences, UFT Tulln, Konrad Lorenz Strasse 24, 3430, Tulln, Austria
}

[3]. Many derivatives have been described and some are still in use today although other binary vectors which are smaller, have a higher copy number, and different selectable markers for use in bacteria (E. coli and Agrobacteria) and in plants have been introduced (for review see [4,5]). One popular series are the pPZP vectors [6] which were also the basis for the pCAMBIA vectors [7]. We have recently published an improved pPZP vector (pPZP3425) which was equipped with a kanamycin resistance gene for selection in Agrobacterium [8]. The strong $35 \mathrm{~S} \mathrm{CaMV}$ promoter driving the plant resistance gene for kanamycin resistance was replaced by the weaker nos promoter because it had been shown that the $35 \mathrm{~S}$ promoter driving the plant resistance marker in the original pPZP vectors can lead to ectopic expression of the transgene $[9,10]$. Furthermore, pPZP3425 contains an expression cassette which consists of an intron-containing GUS gene driven by a strong constitutive promoter (35 S promoter with doubled

\section{Biomed Central}


enhancer plus omega element as translational enhancer). This vector has successfully been used in our laboratory.

Plant selectable markers for the pPZP vectors include kanamycin and gentamycin. Both markers work well for a variety of plant species. Kanamycin is perhaps the most widely used selectable marker for plant transformation. Kanamycin and gentamycin as well as other antibiotic markers have the disadvantage that they are usually used under sterile conditions. In case of Arabidopsis this means that to isolate transgenic plants the seeds have to be sterilized and grown on a sterile agar medium containing the antibiotics. Recently it has been shown that the selection of transgenic plant lines containing a kanamycin marker gene can be done by culturing the seedlings on rockwool saturated with MS medium without sugar but containing the selective agent [11]. Since the medium does not contain sugar, sterile conditions are not necessary, saving costs and labour. However, extreme care has to be taken that the seedlings do not run dry. Other markers that also circumvent the need to work under sterile conditions use resistance against herbicides, especially phosphinotricin (BASTA). The herbicide can be sprayed onto plants growing in soil to select for those containing the Bar gene which mediates resistance against phosphinotricin [12]. Fluorescent proteins have also been reported as markers for plant transformation including Arabidopsis [13-17]. For Arabidopsis transformation, DsRed, GFP, and GFP variants have been used as markers driven by seed-specific promoters derived from other plant species $[18,19]$.

During cloning of a vector for transient expression we realized that the PPZP vectors contain 3 NotI sites in their backbone such that this eight-cutter could not be used in the polylinker. Starting with pPZP200, we have therefore removed all NotI sites from the vector backbone as well as other unnecessary parts to produce pPZP500. By replacing the spectinomycin resistance gene with the nptII gene we also produced the vector pPZP600.

The vector pPZP500 does not contain a plant selectable marker as this is not needed for transient expression. However, since pPZP500 was much smaller than the original pPZP vectors, it could be the basis of a new binary vector (pMAA-Red) for stable transformation of Arabidopsis. For that we included a DsRed gene driven

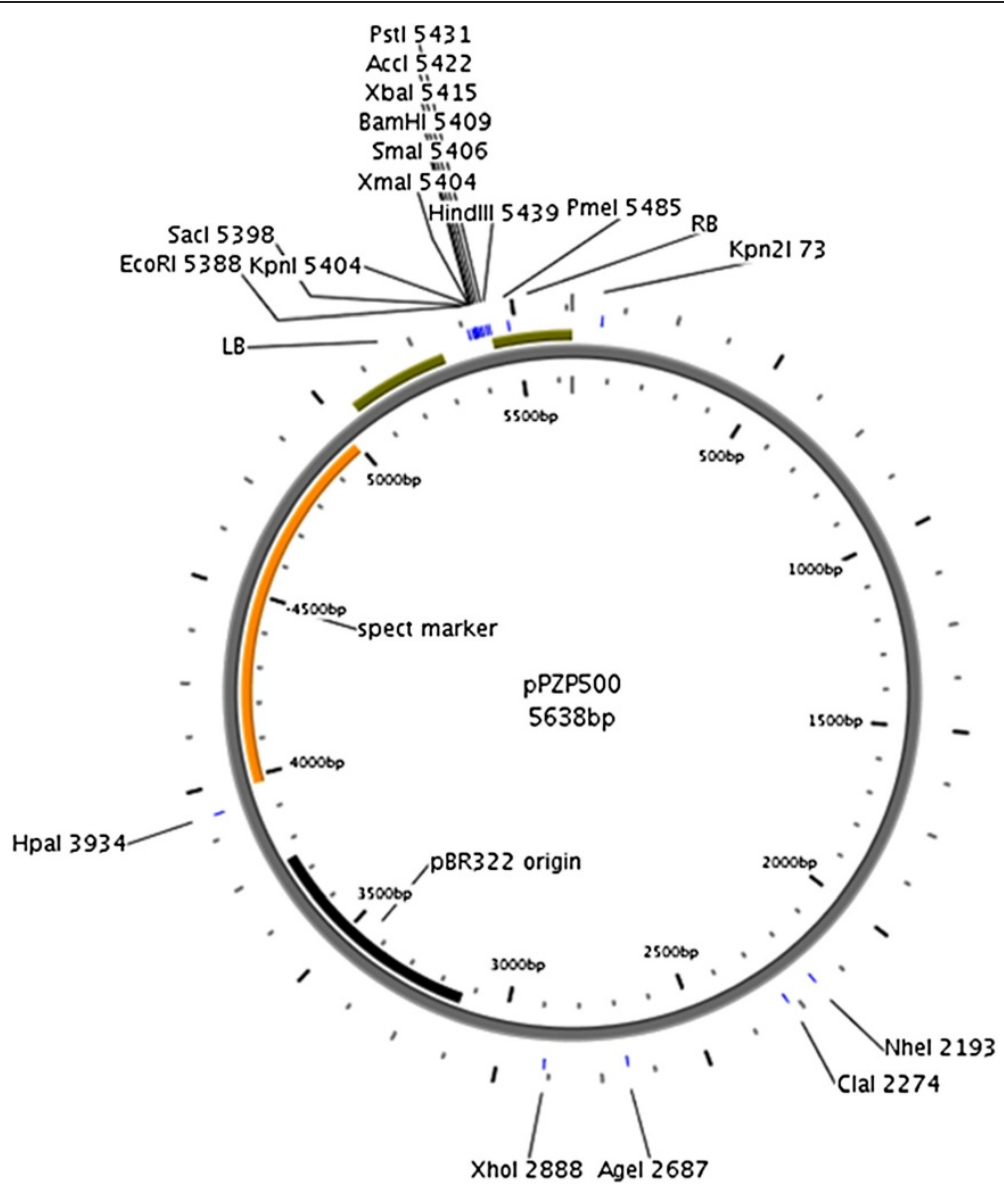

Figure 1 pPZP500. Circular map with main restriction sites, polylinker, and pBR322 origin. LB, left border; RB, right border; spect marker, spectinomycin marker. 
by the Pdf2.1 promoter and the GUS cassette from pPZP3425. The Pdf2.1 promoter was chosen because it is strongly expressed in seeds and in syncytia, feeding sites induced by the beet cyst nematode $H$. schachtii in Arabidopsis roots [20,21]. In addition, we replaced the spectinomycin resistance gene used for selection in Agrobacteria by a kanamycin resistance gene.

\section{Results}

\section{Construction of pPZP500 and pPZP600}

The binary vector pPZP200 is a high copy number, stable, and fully sequenced plasmid vector harbouring the pVSI derived backbone [6]. However, presence of three NotI sites in pPZP200 precluded the use of NotI for cloning. Therefore, these NotI sites have been eliminated by a series of PCR amplifications (Additional file 1: Figure S1) to produce pPZP500 (Figure 1) as described in the Methods section. We confirmed that the vector was still fully functional by introduction of the GUS cassette from pPZP3425 [8] and transient expression of the resulted plasmid pPZP5025 in Nicotiana benthamiana (Figure 2).

Vector pPZP500 contains the streptomycin/spectinomycin resistance gene (aadA, encoding aminoglycoside-3 "-adenyltransferase) which can be used in E. coli and in Agrobacteria. However, in our hands spectinomycin selection was not as tight as kanamycin selection. We, therefore, replaced the spectinomycin resistance gene with the nptII gene which yielded the vector pPZP600 (Additional file 2: Figure S2) as described in Methods.

\section{Construction of pMAA-Red}

Vectors containing a fluorescent protein coupled to a seed-specific promoter have been described before. Here, we have used the fluorescent marker DsRed $[14,22]$ and the promoter of the Pdf2.1 gene [20] from Arabidopsis because we were especially interested in overexpressing genes that are downregulated in syncytia induced by the beet cyst nematode $H$. schachtii in Arabidopsis roots [23]. The reason to use this promoter was that it is not only expressed in seeds but also in syncytia [21]. The Pdf2.1 promoter ( 400 bps) and DsRed region were amplified from the vector pPZP3425-pPDF2.1:: DsRed (Bohlmann, unpublished) which contained a Pdf2.1 promoter fused to DsRed. The Pdf2.1 promoter was amplified using the pPDF2.1EcoFor primer containing an EcoRI site and the pPDF2.1NcoRev primer with NcoI site (Table 1). For amplification of the DsRed $+35 \mathrm{~S}$

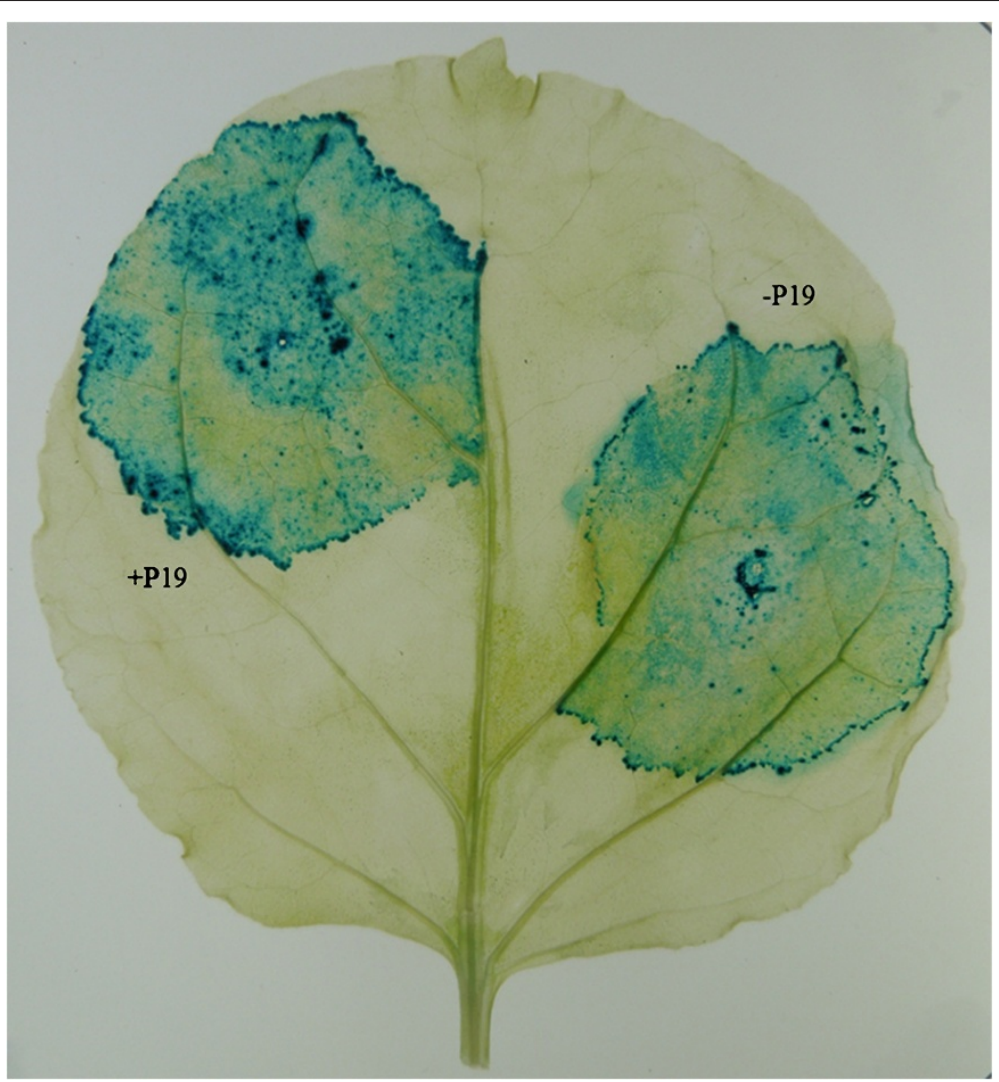

Figure 2 GUS expression from pPZP5025. Nicotiana benthamiana leaves were infiltrated with Agrobacteria harbouring pPZP5025 and stained for GUS expression 5 days later. 
Table 1 List of oligonucleotides used in this work

\begin{tabular}{|c|c|c|}
\hline No & Name & Sequence \\
\hline 1 & PZPN-1/Kpn2lfor & GGCTCCGGAGAATGAACGCCAA \\
\hline 2 & PZPN-1rev & TTCGATCAGCGGTTGCTTGCCA \\
\hline 3 & PZPN-2for & TCGTGGCAAGCAACCGCTGAT \\
\hline 4 & PZPN-2/Xholrev & GCCTCGAGAGGCCGACGCGA \\
\hline 5 & PZPN-1/Kpn2lfor & GGCTCCGGAGAATGAACGCCAA \\
\hline 6 & PZPN-2/Xholrev & GCCTCGAGAGGCCGACGCGA \\
\hline 7 & PZP2/Xholfor & CAGACTCGAGTGTACTGAGAGTGCAC \\
\hline 8 & PZP2/Kpn2Irev & AACGTCCGGAGCCGACTGCACTATAGCA \\
\hline 9 & P500Kp1Nsegfor & ATTCGAGCTCGGTACCCGGG \\
\hline 10 & P500N2segfor & TTAGCGGCTAAAGGAGGCGG \\
\hline 11 & P500Xh3Nsegfor & AGGTCTCTITCCTGTGGATAGC \\
\hline 12 & P500for & GCCCGAGGCATAGACTGTAC \\
\hline 13 & P500rev & GCATCAGACAAACCGGCCAG \\
\hline 14 & PZP500Mphfor & GAATGCATCACAGGCAGCAACGCT \\
\hline 15 & PZP500Mphrev & GCGTGCATAATAAGCCCTACA \\
\hline 16 & KanforMph & CAGCATCATGCATAATTGTGGTTTCA \\
\hline 17 & KanrevMph & GTTGCGATGCATCTAGGTACTAAAACAAT \\
\hline 18 & pPDF2.1EcoFor & AATGAATTCCAGAATGAGTTGTCA \\
\hline 19 & pPDF2.1NcoRev & CATAGAGAACTCCATGGTTGGAGAAAG \\
\hline 20 & RedforBspHI & AGATCATGACCTCCTCCGAGAAC \\
\hline 21 & RedrevBam & AGTCGGATCCGCTAGAGGAACAGGT \\
\hline 22 & DsRedfor2 & CCACCACCTGTTCCTCTAGC \\
\hline 23 & Linker1 & AGCTGAATTCACGTGGTACCGCGGCCGCAGATCTA \\
\hline 24 & Linker2 & AGCTTAGATCTGCGGCCGCGGTACCACGTGAATTC \\
\hline 25 & P500terfor & GCTGGTGGCAGGATATATTG \\
\hline 26 & P500RBseqrev & TTAAACTGAAGGCGGGAAAC \\
\hline 27 & DsRedMfor & CAGAAGAAGGACAATGGGCTGGGA \\
\hline 28 & DsREDnosTERfor & ACCTGTTCCTCTAGGATCGTTTCAAAC \\
\hline 29 & DsREDnosTERrev & TTTGAACGATCCTAGAGGAACAGGTG \\
\hline 30 & nosTERrevEco & TGAGAATTCAGAGATCTAGTAACATAG \\
\hline
\end{tabular}

terminator fragment, Redfor with BspHI and Redrev with BamHI sites were used. The PDF2.1 promoter PCR product was digested with EcoRI and NcoI and that of DsRed with BspHI and BamHI. The vector pPZP600 was digested with EcoRI and BamHI. These three fragments were mixed and ligated together to yield pPZP650 (Additional file 2: Figure S2). The EcoRI and BamHI sites were eliminated sequentially by digestion, filling in the ends using Klenow enzyme, and religation which resulted in the intermediate vector pPZP650.3 (not shown). This intermediate vector was digested with HindIII and a polylinker (PmlI, EcoRI, KpnI, NotI, BglII and HindIII sites provided by oligos Linker1 and 2) was inserted. The orientation of the polylinker was determined by digestion with EcoRI and XbaI (only present in the terminator downstream of the HindIII site). This vector was named pPZP653 (Additional file 2: Figure S2). We then introduced the GUS cassette from PZP3425 by digesting pPZP653 with HindIII and ligating the vector with the GUS cassette derived also by digestion with HindIII to make pPZP6535 (Additional file 2: Figure S2). Orientation of the cassette was tested by PCR using DsRedfor2 and GUSrev primers (Table 1). Since this vector contained two $35 \mathrm{~S}$ terminators we could not use terminator reverse primers for sequencing of our gene of interest delivered by the CaMV promoter. We therefore, replaced the $35 \mathrm{~S}$ terminator in the DsRed cassette with a nos terminator by a series of polymerase chain reactions. In a first step, the last part of DsRed (containing a StuI site) from vector pPZP6535 was amplified by using primers DsRedMfor and DsREDnosTERrev. In the second step, we amplified the nos terminator by using primers DsREDnosTERfor primer and nosTERrevEco (with EcoRI site). Then overlapping PCR was done using DsRedMfor and nosTERrevEco primers. Both, the amplified PCR product and pPZP6535 were digested with StuI and EcoRI and large vector fragment and insert were ligated to produce the final binary vector pMAA-Red (MAA is named after the initials of first author name and Red for Ds-Red) (Figure 3A and B).

\section{Production of transgenic Arabidopsis lines using pMAA- Red}

The vector pMAA-Red was transformed into Agrobacteria and then into Arabidopsis using the floral dip method. Seeds were harvested and red fluorescent seeds were selected using an inverted fluorescence microscope (Figure 4A). These T1 seeds were grown on soil and siliques that showed a 3:1 ratio of fluorescent and nonfluorescent seeds (Figure 4B) were used to produce homozygous lines (Figure 4C and D) which did not show any phenotypic difference. We confirmed the expression of the DsRed cassette in syncytia by growing seedlings from homozygous lines on agar and infection with $H$. schachtii larvae. As expected, in these lines, the Pdf2.1 promoter directed DsRed expression not only in seeds but also in syncytia (Figure 5A and B).

It could be argued that the DsRed expression in syncytia might influence the development of syncytia and lead to higher susceptibility or resistance. To test this possibility, we compared the resistance of an Arabidopsis line transformed with the vector pMAA-Red with wild-type plants at $15 \mathrm{dpi}$. We could not detect significant differences between wild-type plants and the transgenic line as numbers of males and females as well as sizes of syncytia and female nematodes are concerned (Figure 6A and B).

Overexpression lines are often produced to achieve a strong expression of the gene of interest. For that reason strong promoters are used, such as the CaMV $35 \mathrm{~S}$ promoter [24]. However, mainly due to the insertion of the transgene in different parts of the genome, the transgenic lines that are produced vary widely in expression 


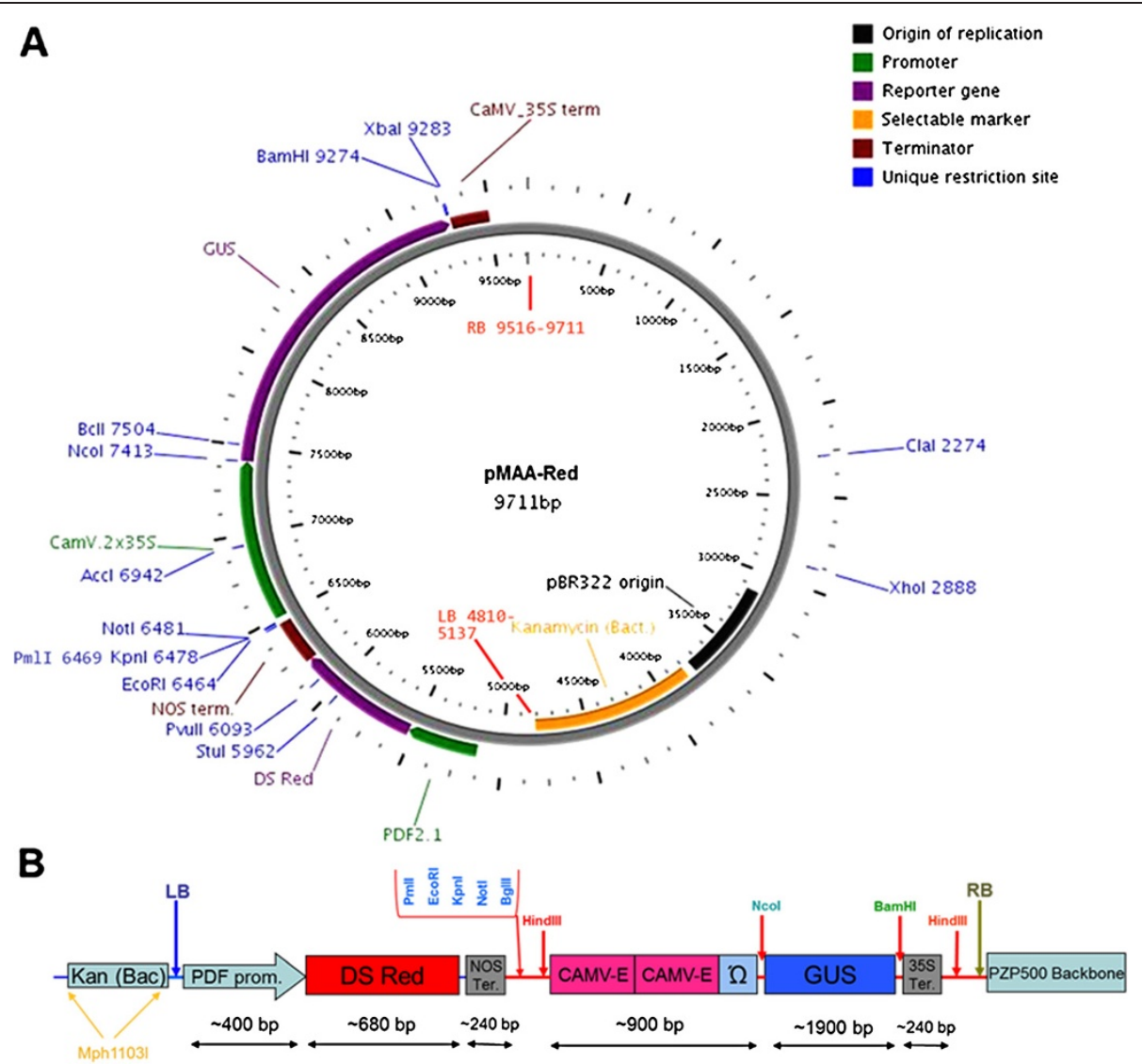

Figure 3 pMAA-Red. Circular map with main restriction sites and polylinker (A), (B) Diagram showing the length of different fragments and restriction sites.
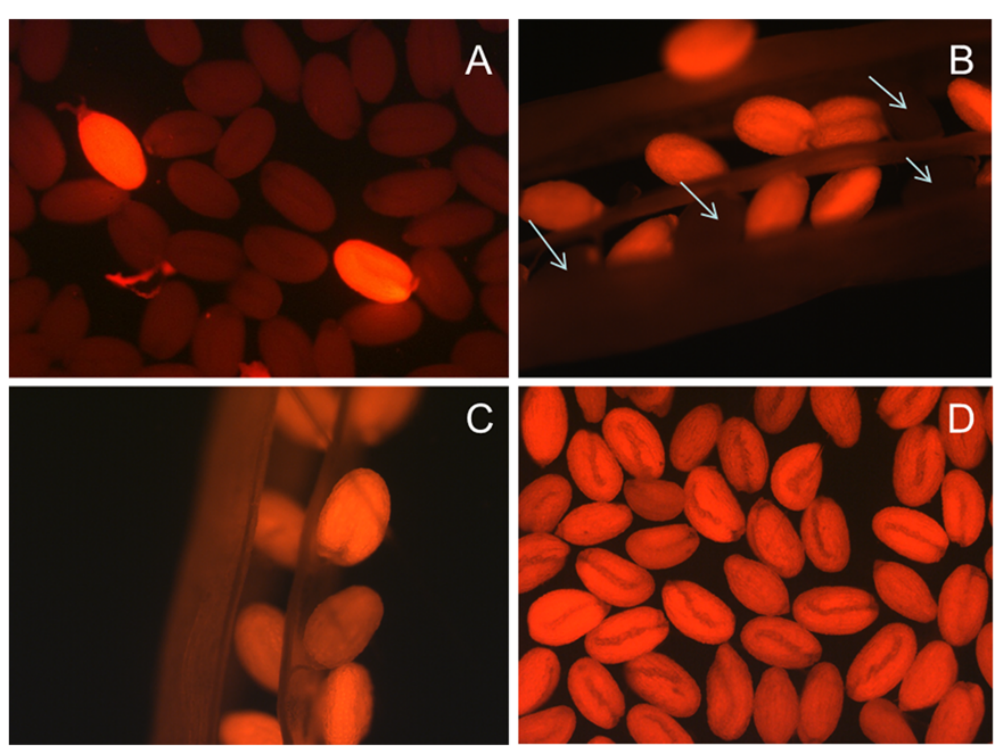

Figure 4 pMAA-Red seed selection. Fluorescent seeds from transgenic Arabidopsis lines transformed with pMAA-Red (A) Selection of transformed seed $\left(T_{0}\right)$ under microscope, (B) Silique in $T_{1}$ generation shows some non-florescent seeds (arrows) representing heterozygous line, (C) Silique in $T_{2}$ generation shows all florescent seeds representing homozygous line, (D) The selected homozygous line in $T_{2}$ generation. 


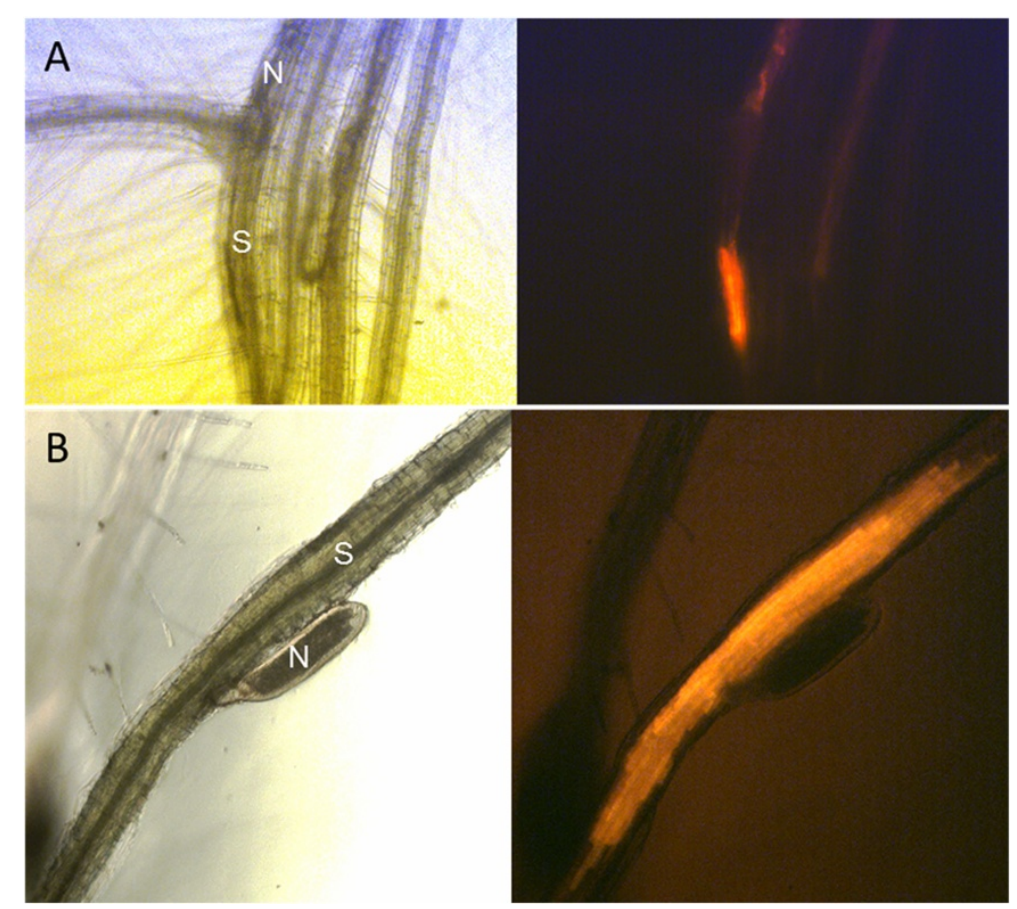

Figure 5 DsRed fluorescence in syncytia. Syncytia induced by $H$. schachtii in roots of an Arabidopsis line transformed with pMAA-Red show DsRed florescence at $5 \mathrm{dpi}(\mathbf{A})$ and $10 \mathrm{dpi}$ (B). Left, bright field image; right DsRed fluorescence.

level [24]. It is therefore necessary to screen a number of lines at the transcript level or the protein level. We reasoned that this lengthy procedure could be shortened if selecting those lines with a strong fluorescence in seeds. To test this hypothesis, we selected 12 transgenic lines transformed with the vector pMAA-Red. We arranged these lines by eye according to the strength of fluorescence in the seeds (Figure 7A). We grew seedlings of these lines and stained the leaves for GUS expression (Figure 7B) which showed a correlation with DsRed expression in seeds. We confirmed this result by measuring the GUS expression level in seedlings (Figure 7C). Thus, to select transgenic lines with a strong expression level, one simply has to select several transgenic seeds with a strong DsRed fluorescence.

\section{Discussion}

A large variety of binary vectors for plant transformation have been described. Among them the pPZP series of vectors [6] and the derived PCAMBIA vectors [7] are especially popular. A prominent feature of these vectors is their stability in bacteria, the high copy number, and the relatively small size. One disadvantage was the use of the CaMV promoter for the plant selectable marker and the use of spectinomycin and chloramphenicol as selectable markers for bacteria which led us to construct the vector pPZP3425 [8]. In this vector the CaMV35S promoter for the plant selectable marker (kanamycin) was replaced by the weaker nos promoter and a kanamycin resistance gene for selection in bacteria was included in the vector backbone.

Although pPZP3425 proved useful for our purposes in producing promoter:: GUS lines (by replacing the CaMV promoter in the GUS cassette with a promoter of interest) or overexpression lines (by replacing GUS in the GUS cassette with a gene of interest) [8], selection of homozygous transgenic lines with a strong expression of the gene of interest was still a lengthy procedure. Since we were interested to produce a large number of transgenic Arabidopsis overexpression lines with a strong expression level for putative antimicrobial peptides we set out to construct a pPZP vector that would have three important features: First, it should allow us to use selection or screening on soil to avoid growing Arabidopsis under sterile conditions. Second, we wanted to easily select lines with a strong expression level. Third, the vector should be used for the expression in syncytia using specific promoters that would not be active in seedlings or leaves.

The first precondition excluded the use of antibiotic resistance such as kanamycin or hygromycin or the use of metabolite resistance genes such as the $E$. coliderived phosphomannose isomerase which allows growth on mannose [25] or the Streptomyces rubiginosus xylose isomerase $(x y l A)$ gene which allows growth on xylose [26] as a plant selectable marker for which sterile conditions would have to be used. Herbicide resistance would 


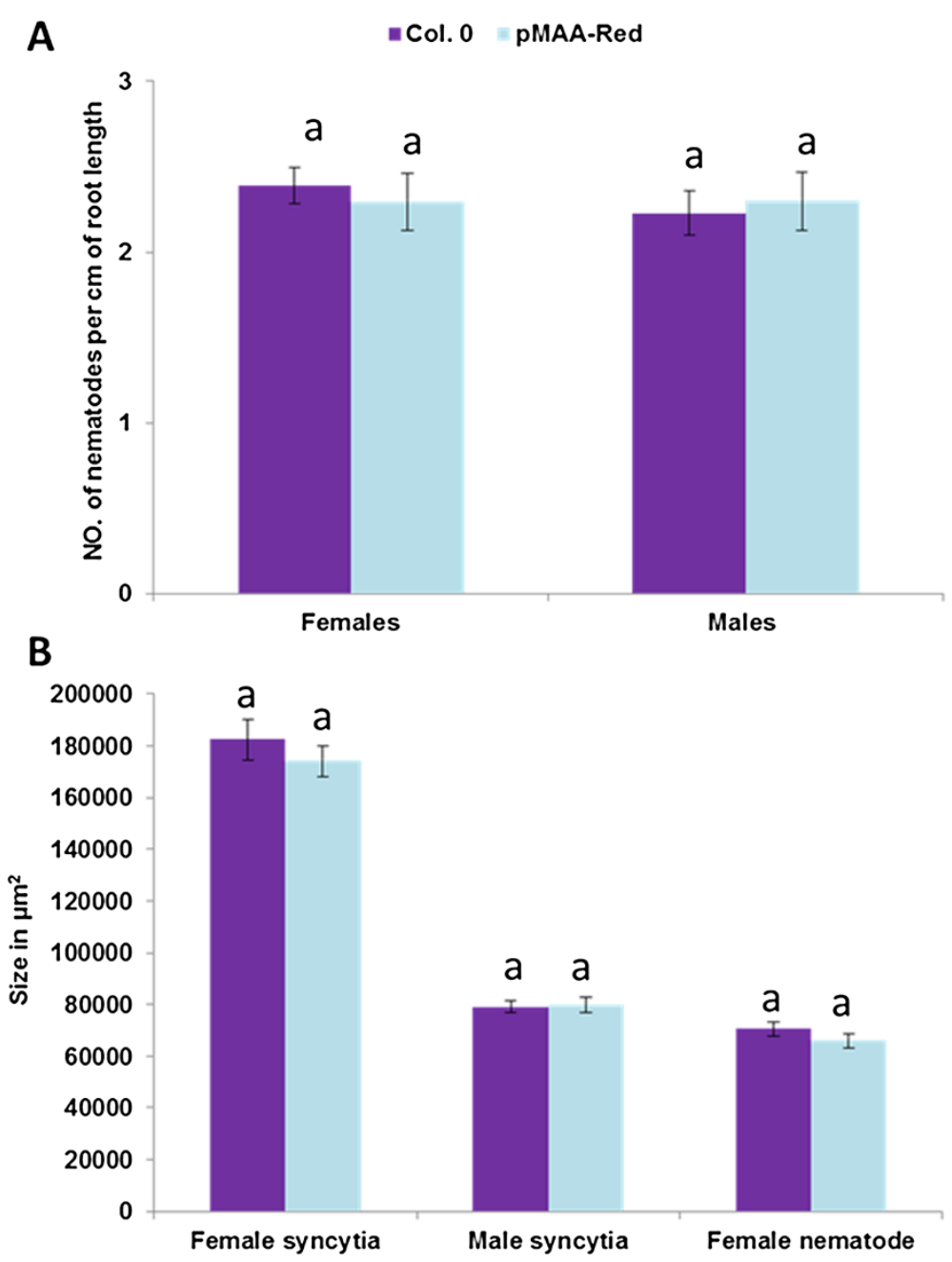

Figure 6 Nematode infection test. Infection assay of a homozygous line transformed with pMAA-Red compared with wild-type (Col.0) plants. (A) Number of male and female nematodes per $\mathrm{cm}$ of root was determined at $15 \mathrm{dpi}$ showing no significant differences between wild-type and the vector line $(P<0.05$, one way ANOVA and LSD). The statistical significance was determined from three independent experiments each having 5 plates with 10 plants per plate. Values are means $\pm S E, n=15$. (B) Size of female syncytia, male syncytia, and female nematodes at 15 dpi was not different between the vector line and the wild-type $(P<0.05$, one way ANOVA and LSD). Values are means $\pm S E, n=30$.

avoid the need for sterile growth but all T1 seedlings have to be grown for selection. In contrast, if using a fluorescent marker that is expressed in seeds, only the transgenic seeds obtained after transformation would have to be grown further and no additional treatment would be needed. Such an approach is especially important if a large number of transgenic lines have to be produced. An example is the work of [27] who conducted a highthroughput screen in Arabidopsis for castor genes that would lead to changes in hydroxy fatty acid composition in seeds. We decided to try the DsRed gene as a screenable marker [13-17]. This leaves the possibility to use in addition GFP or other most frequently used fluorescent markers as reporter genes. Furthermore, DsRed has a rather weak fluorescence which might seem to be a disadvantage. However, this weak fluorescence makes it easier to identify seeds with a different level of fluorescence.

Expression of the gene of interest varies largely in independent transgenic lines [24] due mainly to position effects. One way of reducing this variation is the inclusion of matrix attachment regions in the T-DNA. However, this only worked with gene silencing mutants, which limits the use of these vectors [28]. We have demonstrated here that the use of the vector pMAARed allows an easy and efficient selection of transgenic lines with a strong expression of the gene of interest in wild-type Arabidopsis plants. Of course, mutants could also be used, if needed. Pre-selection of lines with a strong DsRed expression in seeds according to their fluorescence reduces the number of lines that have to be tested at the transcript or protein level. 
A

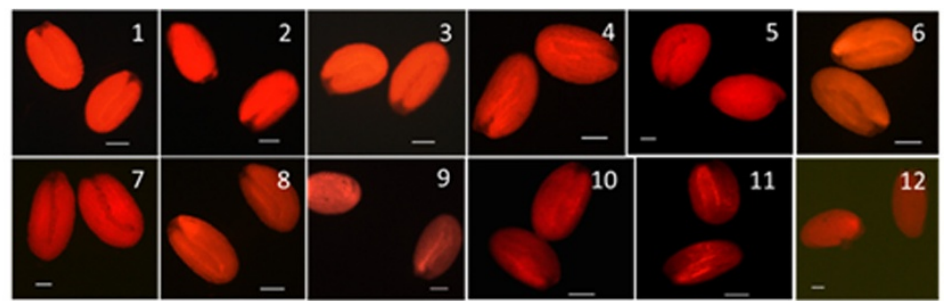

B

12

3

4

$5 \quad 6$
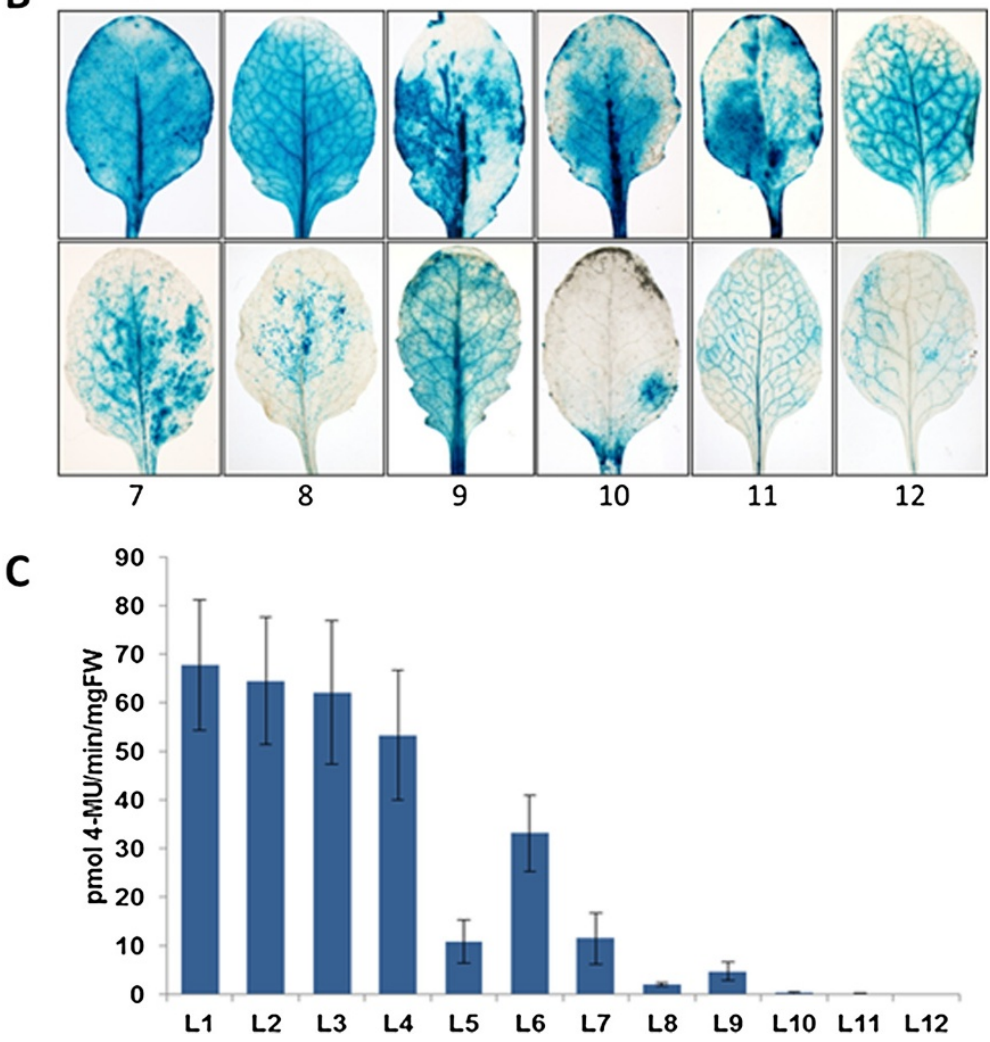

Figure 7 Selection of lines with a strong expression level. Seeds from 12 transgenic lines of pMAA-Red were arranged according to decreasing fluorescence (A). Histochemical GUS staining of leaves from these lines is shown in (B) and GUS quantification of seedlings in (C).

Our third precondition was that the vector should allow the use of syncytium-specific promoters instead of the CaMV promoter which is active in most tissues of Arabidopsis plants. We have recently shown that several genes are expressed in syncytia which are normally expressed in pollen [23], such as MIOX4 and MIOX5, or in seeds, such as Pdf2.1 whose promoter is used here to drive the expression of DsRed in seeds. Using the MIOX4 or MIOX5 promoter would allow a specific expression in syncytia which could be useful for genes with a negative effect on plant growth. However, screening such transgenic lines would require the analysis of expression of the gene of interest in syncytia. Such a screening is very time consuming because syncytia have to be cut out from infected roots. In this case a pre-screening that would reduce the number of lines would lead to a significant reduction of time and effort. Thus, the pMAA-Red vector that we have constructed is especially useful for the syncytium-specific expression of transgenes or for similar cases where the expression of the transgene would be restricted to tissues that could not be easily screened. Promoter::reporter constructs and overexpression lines can be produced from this vector as from pPZP3425 [8]. The CaMV promoter can be replaced by a promoter of interest using NcoI and one of the unique sites in the polylinker. The GUS sequence can be replaced with a sequence for overexpression of a gene of interest by using NcoI and BamHI. If the sequence to be cloned contains NcoI or BamHI site, it is usually possible to use a restriction enzyme that produces compatible cohesive ends with NcoI and BamHI. For instance, BspHI, PciI, and FatI produce cohesive ends that are compatible with NcoI.

After transformation of Arabidopsis plants it takes about 3-4 weeks until T1 seeds can be harvested and 
inspected for fluorescent seeds. A big advantage of transformation with the vector pMAA-Red is that only these selected seeds have to be grown on soil to produce the T2 generation. After another 4 weeks, the first siliques of these plants can be screened for a 3:1 segregation of fluorescent seeds which can then be used to produce homozygous T3 seeds for further analysis. Again, the first siliques of these plants can be used to select homozygous lines and only those will be grown for maturity, which will take a total of 6 to 8 weeks. Depending on the growth conditions, the whole procedure from transformation to harvesting homozygous seeds could be completed within four month.

\section{Conclusion}

We have constructed compact pPZP vectors without NotI sites having either bacterial spectinomycin or kanamycin resistance (pPZP500 and pPZP600, respectively) and a vector (pMAA-Red) which allows an easy production of transgenic Arabidopsis overexpression lines with strong expression levels of the gene of interest.

\section{Methods}

\section{Cloning methods}

Standard procedures were used for restriction enzyme mediated DNA digestion, ligation, and transformation [29]. Restriction enzymes and T4 DNA ligase were from Fermentas -Thermo Fisher Scientific and New England Biolabs. The $E$ coli strain DH10B was used throughout this work. PCR was done using Eppendorf Mastercycler Gradient.

\section{Construction of pPZP500}

The NotI sites in pPZP200 were eliminated by a series of PCR amplifications using pPZP200 as template. Ligation of the amplified fragments yielded a binary vector without NotI sites. The outline of this construction and the position of primers is given in Additional file 1: Figure S1. In a first round of PCR reactions, a 1549 bp backbone fragment from pPZP200 was amplified using primers PZPN-1 /Kpn2Ifor and PZPN-1rev (see Table 1 for primers and Table 2 for vectors used in this work) and a 1283 bp fragment was amplified by using primers PZPN-2for and PZPN-2/XhoIrev. Both fragments were mixed and used as template in a second PCR to amplify a 2824 bp fragment (first half of pPZP200 i.e., so called insert) by using primers PZPN-1/Kpn2Ifor and PZPN-2/XhoIrev. In another PCR reaction the remaining half $(2814 \mathrm{bp})$ of pPZP200 (i.e., so called vector) was amplified by using primers PZP2/XhoIfor and PZP2/Kpn2Irev. Vector and insert fragment were digested with Kpn2I and XhoI and ligated to yield pPZP500 (Figure 1) that was transformed in E. coli for selection purpose and the modified parts were sequenced using primers P500Kp1Nseqfor, P500N2seqfor, P500Xh3Nseqfor, P500for, and P500rev. To further confirm that after removing some sites from the backbone of pPZP200 the new pPZP500 vector is functional, we have cloned a GUS cassette (containing a duplicated CaMV $35 \mathrm{~S}$ promoter with omega element, uidA gene with intron, and CaMV terminator) from pPZP3425 [8] as HindIII fragment into the same site of pPZP500 to produce pPZP5025 (Additional file 2: Figure S2). This plasmid was transformed into Agrobacteria which were used for agroinfiltration into leaves of $N$. benthamiana. Four days after infiltration, leaves were processed for GUS staining, indicating full functionality of the vector (Figure 2).

\section{Construction of pPZP600}

The spectinomycin resistance gene of pPZP500 was replaced by the nptII gene. The nptII gene $(\sim 1 \mathrm{~kb})$ was amplified from pPZP3425 using KanforMph and KanrevMph primers. The pPZP500 vector backbone was amplified by using PZP500Mphfor and PZP500Mphrev primers. Both primers had a Mph1103I site at their ends. Insert and vector fragment were digested with Mph1103I, ligated, and transformed into E. coli to yield pPZP600 (Additional file 2: Figure S2).

\section{Table 2 Vectors used in this study}

\begin{tabular}{|c|c|c|c|}
\hline No & Name & Features & Reference \\
\hline 1 & pPZP200 (Additional file 1: Figure S1) & Spectinomycin resistance gene for bacterial slection & {$[6]$} \\
\hline 2 & pPZP3425 & $\begin{array}{l}\text { CaMV } 35 \text { S } 2 \text { X promoter with omega element, LacZ operon, GUS, } \\
\text { CaMV terminator (GUS cassette), kanamycin resistance gene for } \\
\text { bacterial and plant selection }\end{array}$ & {$[8]$} \\
\hline 3 & pPZP500 (Figure 1) & pPZP200 modified backbone without Notl sites & This study \\
\hline 4 & pPZP5025 (Additional file 2: Figure S2) & pPZP500 with GUS cassette & This study \\
\hline 5 & pPZP3425-pPDF2.1::DsRed & pPZP3425 containing pPDF2.1::DsRed instead of the 35 S::GUS cassette & Bohlmann, unpublished \\
\hline 6 & pPZP600 (Additional file 2: Figure S2) & pPZP500 with kanamycin resistance gene for bacterial selection & This study \\
\hline 7 & pPZP650 (Additional file 2: Figure S2) & pPZP600 with PDF2.1 promoter, DsRed gene, and 35 S terminator & This study \\
\hline 8 & pPZP653 (Additional file 2: Figure S2) & pPZP650 with new polylinker & This study \\
\hline 9 & pPZP6535 (Additional file 2: Figure S2) & pPZP653 with GUS cassette & This study \\
\hline 10 & pMAA-Red (Figure 3) & pPZP6535 with PDF2.1 promoter, DsRed gene, nos terminator and GUS cassette & This study \\
\hline
\end{tabular}




\section{Plant growth}

$N$. benthamiana and Arabidopsis plants were grown in a growth chamber at $25 \pm 1^{\circ} \mathrm{C}$, with a 16 hour light/ 8 hour dark photoperiod and approximately $65 \%$ humidity.

\section{Agrobacterium transformation}

Binary plasmids were transformed into A. tumifaciens strain GV3101 by the freeze-thaw method [30]. Agrobacteria were selected on YEB plates with appropriate antibiotics which included $25 \mu \mathrm{g} / \mathrm{ml}$ gentamycin and $35 \mu \mathrm{g} / \mathrm{ml}$ rifampicin for Agrobacteria. Selection for binary plasmids was done with $200 \mu \mathrm{g} / \mathrm{ml}$ spectinomycin (for pPZP5025) or $50 \mu \mathrm{g} / \mathrm{ml}$ kanamycin (for pMAA-Red).

\section{Agroinfiltration of N. Benthamiana}

Agrobacteria were grown to an $\mathrm{OD}_{600}$ of 0.8 overnight in an incubator/shaker at $28^{\circ} \mathrm{C}$. Bacteria were harvested by centrifugation at $5000 \mathrm{rpm}$ for $6 \mathrm{~min}$ in a table top centrifuge at room temperature and suspended in infiltration medium (10 mM MES pH 5.6, $10 \mathrm{mM} \mathrm{MgCl}_{2}$ and $100 \mu \mathrm{M}$ acetosyringone) to obtain bacterial suspensions of the an $\mathrm{OD}_{600}$ of 1.0. After incubation for $2 \mathrm{hr}$ at room temperature, Agrobacterium suspensions were infiltrated in the abaxial side of leaves by using a $1 \mathrm{ml}$ syringe without a needle. Infiltrated plants were kept under the same growth conditions as mentioned above. For co-infiltration of the RNA silencing inhibitor P19, an equal volume of a bacterial suspension harbouring pBin61P19 [31] was added prior to infiltration.

\section{Arabidopsis transformation}

The vector construct pMAA-Red was transformed into Arabidopsis ecotype Col by the floral dip method [32]. Seeds of transformed plants were harvested and photographed under an inverse microscope equipped with a DsRed fluorescence filter (Axiovert 200 M; Zeiss, Hallerbergmoos, Germany) and an integrated camera (AxioCam MRc5; Zeiss). Fluorescent seeds $\left(T_{1}\right)$ were selected and put on soil to grow the next generation $\left(\mathrm{T}_{2}\right)$, of which the first mature silique was detached from the plant and examined under the microscope for fluorescent seeds to check for 3:1 segregation. These seeds were then used to produce homozygous lines. Seeds that were kept in the dark at $4^{\circ} \mathrm{C}$ still showed fluorescence after several years.

\section{GUS staining}

GUS staining of the $N$. benthamiana leaves was done by overnight incubation in X-Gluc solution $(50 \mathrm{mM}$ sodium phosphate buffer pH 7.0, 10 mM EDTA pH 8.0, 0.1\% (v/v) Triton X-100 and $0.5 \mathrm{mg} / \mathrm{ml} \mathrm{X-Gluc)}$ at $37^{\circ} \mathrm{C}$ [33] followed by several washings with $70 \%$ ethanol to remove the chlorophyll from leaf tissues.

\section{GUS activity assay}

GUS activity was measured according to Jefferson et al. [33] with some modifications in black 96-well Greiner plates. To $100 \mu \mathrm{l}$ of total protein extract $50 \mu \mathrm{l}$ of $4 \mathrm{mM}$ 4-MUG was added. The reaction was incubated at $37^{\circ} \mathrm{C}$ for $5 \mathrm{~min}$ and then $50 \mu \mathrm{l}$ of $0.5 \mathrm{M} \mathrm{Na}_{2} \mathrm{CO}_{3}$ was added to stop the reaction. Fluorescence was measured at $355 \mathrm{~nm}$ excitation and $460 \mathrm{~nm}$ emission in a FLUOstar Omega micro plate reader (BMG Labtech) using 4-MU standards $(10 \mathrm{mM}$ stock in ethanol and diluted in GUS extraction buffer) in the range of $1-100 \mu \mathrm{M}$.

\section{Nematode infection and examination of fluorescence in syncytia}

The seeds of a pMAA-Red transgenic Arabidopsis line were surface-sterilized for $7 \mathrm{~min}$ in 10\% Chlorox (v/v), submerged for $5 \mathrm{~min}$ in $70 \%(\mathrm{v} / \mathrm{v})$ ethanol, and then washed three times in sterile water. The sterilized seeds were placed on a modified 0.2 concentrated Knop agar medium supplemented with $2 \%$ sucrose [34]. H. schachtii was multiplied in vitro on mustard (Sinapsis alba cv. Albatros) roots growing on 0.2 concentrated Knop medium supplemented with $2 \%$ sucrose [34]. Hatching of $\mathrm{J}_{2}$ larvae was stimulated by soaking the cysts in sterile $3 \mathrm{mM} \mathrm{ZnCl}_{2}$. The juveniles were washed four times in sterile water and resuspended in $0.5 \%(\mathrm{w} / \mathrm{v})$ Gelrite for inoculation. Roots of 12-day-old Arabidopsis plants were inoculated with about 40-50 juveniles under sterile conditions. At 5 and 10 dpi the fluorescent syncytia were examined using an inverse microscope.

\section{Resistance tests and size measurement of syncytia and nematodes}

H. schachtii (Schmidt) infection was done in the same way as described above. Roots of 12-day old Arabidopsis plants were inoculated under axenic conditions with about 50 juveniles per plant. The number of male and female nematodes per $\mathrm{cm}$ of root length were counted at $14 \mathrm{dpi}$. The data were analysed using single factor ANOVA $(\mathrm{P}<0.05)$. As the Fstatistic was greater than F-critical, a Least Significance Test (LSD) was applied. At $14 \mathrm{dpi}$, pictures of male syncytia, female syncytia and females nematodes (30 photos for each) were taken. Syncytia and nematodes were measured using an inverse microscope (Axiovert $200 \mathrm{M}$; Zeiss, Hallerbergmoos, Germany). The data were analyzed using single factor ANOVA (P<0.05).

\section{Additional files}

Additional file 1: Figure S1. Position of Primers forNotl Elimination in pPZP200.

Additional file 2: Figure S2. The various intermediate constructs made during the construction of pMAA-Red from pPZP600. 


\section{Competing interests}

The authors declare that they have no competing interests.

\section{Authors' contributions}

MAA cloned pMAA-Red and did the nematode experiments. KHS cloned pPZP500 and did the GUS assays. MAA and KHS helped to draft the manuscript. HB conceived and coordinated the study and helped in writing the manuscript. All authors read and approved the final manuscript.

\section{GenBank accession numbers}

PPZP500: JX025644

pPZP600: JX025643

pMAA-Red: JX025642

\section{Acknowledgements}

This research was supported by grants P20471-B11 and P21896-B16 of the Austrian Science Fund (FWF). Muhammad Amjad Ali and Kausar Hussain Shah were supported by Higher Education Commission (HEC) of Pakistan. The authors are thankful to Shahid Siddique, Amjad Abbas, Bachar Almaghrabi, Martina Niese and Stephan Plattner for their support. We thank Dr. David Baulcombe for pBin61P19.

Received: 16 March 2012 Accepted: 1 June 2012

Published: 2 July 2012

\section{References}

1. Wang K (Ed): Agrobacterium Protocols. 2nd edition. Totowa, New Jersey: Humana Press; 2006

2. Fraley RT, Rogers SG, Horsch RB: Genetic-Transformation in Higher-Plants. Crit Rev Plant Sci 1986, 4(1):1-46.

3. Bevan M: Binary Agrobacterium vectors for plant transformation. Nucleic Acids Res 1984, 12(22):8711-8721.

4. Lee LY, Gelvin SB: T-DNA binary vectors and systems. Plant Physio/ 2008, 146(2):325-332.

5. Komori T, Imayama T, Kato N, Ishida Y, Ueki J, Komari T: Current status of binary vectors and superbinary vectors. Plant Physiol 2007,

145(4):1155-1160.

6. Hajdukiewicz P, Svab Z, Maliga P: The Small, Versatile Ppzp Family of Agrobacterium Binary Vectors for Plant Transformation. Plant Mol Biol 1994, 25(6):989-994.

7. pCambia Vectors.; http://www.cambia.org/daisy/cambia/585.

8. Szakasits D, Siddique S, Bohlmann H: An improved pPZP vector for Agrobacterium-mediated plant transformation. Plant Mol Biol Rep 2007, 25(3-4):115-120.

9. Yang W, Jefferson RA, Huttner E, Moore JM, Gagliano WB, Grossniklaus U: An egg apparatus-specific enhancer of Arabidopsis, identified by enhancer detection. Plant Physiol

2005, 139(3):1421-1432.

10. Yoo SY, Bomblies K, Yoo SK, Yang JW, Choi MS, Lee JS, Weigel D, Ahn JH: The $35 \mathrm{~S}$ promoter used in a selectable marker gene of a plant transformation vector affects the expression of the transgene. Planta 2005, 221(4):523-530.

11. Hadi MZ KE, Wendeler E, Reiss B: Simple and versatile selection of Arabidopsis transformants. Plant Cell Rep 135, 21:130.

12. Dhalluin K, Deblock M, Denecke J, Janssens J, Leemans J, Reynaerts A, Botterman J: The BAR gene as selectable and screenable marker in plantengineering. Methods Enzymol 1992, 216:415-426.

13. Wenck A, Pugieux C, Turner M, Dunn M, Stacy C, Tiozzo A, Dunder E, van Grinsven E, Khan R, Sigareva M, et al: Reef-coral proteins as visual, nondestructive reporters for plant transformation. Plant Cell Rep 2003, 22(4):244-251.

14. Baird GS, Zacharias DA, Tsien RY: Biochemistry, mutagenesis, and oligomerization of DsRed, a red fluorescent protein from coral. Proc Natl Acad Sci USA 2000, 97(22):11984-11989.

15. Tzfira T, Tian GW, Lacroix B, Vyas S, Li JX, Leitner-Dagan Y, Krichevsky A, Taylor T, Vainstein A, Citovsky V: pSAT vectors: a modular series of plasmids for autofluorescent protein tagging and expression of multiple genes in plants. Plant Mol Biol

2005, 57(4):503-516.
16. Lin M-H, Gresshoff PM, Indrasumunar A, Ferguson BJ: pHairyRed: A Novel Binary Vector Containing the DsRed2 Reporter Gene for Visual Selection of Transgenic Hairy Roots. Molecular Plant 2011, 4(3):537-545

17. Ckurshumova W, Caragea AE, Goldstein RS, Berleth T: Glow in the Dark: Fluorescent Proteins as Cell and Tissue-Specific Markers in Plants. Molecular Plant 2011, 4(5):794-804.

18. Goodin MM, Dietzgen RG, Schichnes D, Ruzin S, Jackson AO: pGD vectors: versatile tools for the expression of green and red fluorescent protein fusions in agroinfiltrated plant leaves. Plant J2002, 31(3):375-383.

19. Matz MV: Fluorescent proteins from nonbioluminescent Anthozoa species (vol 17, pg 969, 1999). Nat Biotechnol 1999, 17(12):1227-1227.

20. Thomma BPHJ, Broekaert WF: Tissue-specific expression of plant defensin genes PDF2.1 and PDF2.2 in Arabidopsis thaliana. Plant Physiol Biochem 1998, 36(7):533-537.

21. Siddique S, Wieczorek K, Szakasits D, Kreil DP, Bohlmann H: The promoter of a plant defensin gene directs specific expression in nematodeinduced syncytia in Arabidopsis roots. Plant Physiol and Biochem 2011, 49(10):1100-1107.

22. Jach $G$, Binot E, Frings S, Luxa K, Schell J: Use of red fluorescent protein from Discosoma sp (dsRED) as a reporter for plant gene expression. Plant J 2001, 28(4):483-491.

23. Szakasits D, Heinen P, Wieczorek K, Hofmann J, Wagner F, Kreil DP, Sykacek $P$, Grundler FMW, Bohlmann H: The transcriptome of syncytia induced by the cyst nematode Heterodera schachtii in Arabidopsis roots. Plant J 2009, 57(5):771-784.

24. Holtorf S, Apel K, Bohlmann H: Comparison of different constitutive and inducible promoters for the overexpression of transgenes in Arabidopsis thaliana. Plant $\mathrm{Mol} \mathrm{Biol}$

1995, 29(4):637-646.

25. Weisser P, Kramer R, Sprenger GA: Expression of the Escherichia coli pmi gene, encoding phosphomannose-isomerase in Zymomonas mobilis, leads to utilization of mannose as a novel growth substrate, which can be used as a selective marker. Appl Environ Microb 1996, 62:4155-4161.

26. Haldrup A, Noerremark M, Okkels F: Plant selection principle based on xylose isomerase. In Vitro Cellular \& Developmental Biology-Plant 2001, 37(2):114-119.

27. Lu C, Fulda M, Wallis J, Browse J: A high-throughput screen for genes from castor that boost hydroxy fatty acid accumulation in seed oils of transgenic Arabidopsis. Plant $J$ 2006, 45(5):847-856.

28. Butaye K, Goderis I, Wouters P, Pues J, Delaure S, Broekaert W, Depicker A, Cammue B, De Bolle M: Stable high-level transgene expression in Arabidopsis thaliana using gene silencing mutants and matrix attachment regions. Plant $J$ 2004, 39(3):440-449

29. Sambrook J, Fritsch EF, Maniatis T: Molecular Cloning: A Laboratory Manual. NY: Cold Spring Harbor Laboratory Press; 1989. 1, 2, 3.

30. Holsters M, Dewaele D, Depicker A, Messens E, Vanmontagu M, Schell J: Transfection and transformation of Agrobacterium tumefaciens. Mol Gen Genet 1978, 163(2):181-187.

31. Voinnet $O$, Rivas $S$, Mestre $P$, Baulcombe D: An enhanced transient expression system in plants based on suppression of gene silencing by the 19 protein of tomato bushy stunt virus. Plant $J$ 2003, 33(5):949-956.

32. Logemann EBR, Ülker B, Somssich IE: An improved method for preparing Agrobacterium cells that simplifies the Arabidopsis transformation protocol. Plant Methods 2006, 2(16).

33. Jefferson RA, Kavanagh TA, Bevan MW: Gus Fusions - Beta-Glucuronidase as a Sensitive and Versatile Gene Fusion Marker in Higher-Plants. EMBO J 1987, 6(13):3901-3907.

34. Sijmons PC, Grundler FMW, Vonmende N, Burrows PR, Wyss U: ArabidopsisThaliana as a New Model Host for Plant-Parasitic Nematodes. Plant J 1991, 1(2):245-254.

doi:10.1186/1472-6750-12-37

Cite this article as: Ali et al:: pMAA-Red: a new pPZP-derived vector for fast visual screening of transgenic Arabidopsis plants at the seed stage. BMC Biotechnology 2012 12:37. 